\title{
Effect of a Common Genetic Variant (p.V444A) in the Bile Salt Export Pump on the Inhibition of Bile Acid Transport by Cholestatic Medications
}

\author{
Izna Ali ${ }^{1}$, Seher Khalid ${ }^{1}$, Bruno Stieger ${ }^{2}$, and Kim L.R. Brouwer ${ }^{1, *}$ \\ ${ }^{1}$ Division of Pharmacotherapy and Experimental Therapeutics, UNC Eshelman School of \\ Pharmacy, University of North Carolina, Chapel Hill, North Carolina, USA. ${ }^{2}$ Department of Clinical \\ Pharmacology and Toxicology, University Hospital Zurich, University of Zurich, Zurich, \\ Switzerland.
}

\begin{abstract}
The bile salt export pump (BSEP) is the primary canalicular transporter responsible for the secretion of bile acids from hepatocytes into bile canaliculi, and inhibition of this transporter has been associated with drug-induced liver injury (DILI). A common variant (rs2287622; p.V444A) in the gene encoding for BSEP has been associated with increased risk of cholestatic DILI. Although p.444V BSEP (reference) and p.444A BSEP (variant) do not differ in the transport kinetics of TCA, transport of the more abundant GCA has not been investigated. Importantly, differences in susceptibility of p.444V and p.444A BSEP to inhibition by drugs causing cholestatic DILI has not been investigated. To address these issues, the transport kinetics of GCA were evaluated by incubating membrane vesicles expressing either p.444V or p.444A BSEP with GCA over a range of concentrations $(1,10,25,50,100 \mu \mathrm{M})$. The ability of commonly used cholestatic medications to inhibit transport of TCA and GCA by the reference and variant proteins were compared. Resulting data indicated that GCA transport kinetics for reference and variant BSEP followed Michaelis-Menten kinetics and were not statistically different $\left(\mathrm{V}_{\max }: 1132 \pm 246\right.$ vs. $959 \pm 256 \mathrm{pmol} / \mathrm{min} / \mathrm{mg}$ protein, respectively; $\mathrm{K}_{\mathrm{m}}: 32.7 \pm 18.2$ vs. $45.7 \pm 25.5 \mu \mathrm{M}$, respectively). There were no statistically significant differences between the reference and variant BSEP in the inhibition of TCA or GCA transport by the cholestatic drugs tested. In conclusion, an association between the variant BSEP and risk for cholestatic DILI due to the drugs tested cannot be accounted for by differential inhibition of TCA or GCA transport.
\end{abstract}

\section{Graphical Abastract}

*Corresponding Author: KLR Brouwer (kbrouwer@unc.edu), CB \#7569 Kerr Hall, Chapel Hill, NC 27599-7569, Phone: (919) 962-7030, FAX: (919) 962-0644. 


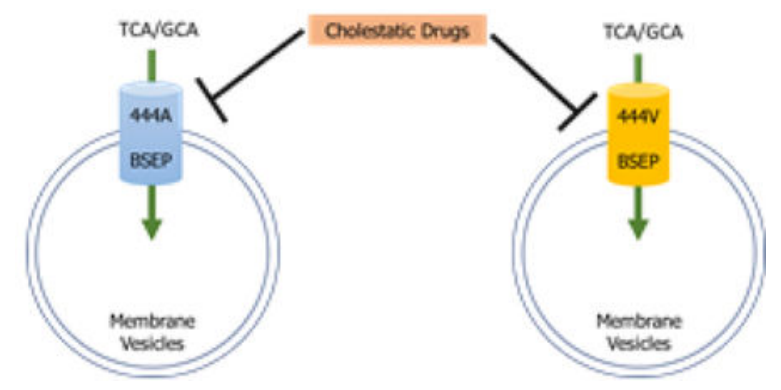

\section{Keywords}

transporters; cholestasis; bile salt export pump; drug-induced liver injury

\section{Introduction}

Transporters play an essential role in the disposition of both medications and endogenous substrates, including bile acids. Bile acids are key metabolic regulators that are synthesized in the liver from cholesterol and are important for the intestinal absorption of dietary lipids. ${ }^{1}$ The disposition of bile acids is tightly regulated because accumulation of these detergentlike molecules in hepatocytes can lead to cellular damage and cholestatic injury. ${ }^{2}$ Bile acids are secreted from the hepatocyte into the bile canaliculi by bile salt export pump (BSEP), an ATP binding cassette (ABC) transporter. ${ }^{3,4}$ The physiological importance of BSEP is demonstrated by inherited cholestatic conditions caused by genetic mutations in $A B C B 11$, the gene encoding for BSEP. These conditions range in severity from those leading to progressive and persistent cholestasis requiring liver transplantation (as seen with progressive familial intrahepatic cholestasis (PFIC) Type II), to milder, self-limiting forms of cholestasis (reported in patients with benign recurrent intrahepatic cholestasis (BRIC)). 5,6 Genetic variants in $A B C B 11$ have been implicated as a potential contributing factor to the development of intrahepatic cholestasis of pregnancy (ICP). In ICP, pregnancy hormones precipitate the development of cholestasis, typically during the second trimester of pregnancy when estrogen levels are highest, consistent with the finding that these hormones alter bile acid disposition. ${ }^{7,8}$ In addition, the inhibition of BSEP by medications can lead to the accumulation of bile acids in the hepatocyte and contribute to the development of cholestatic drug-induced liver injury (DILI). ${ }^{9,10}$

DILI is a major, albeit rare, safety concern for both currently approved mediations and those in the drug development pipeline. DILI may lead to black-box warnings for currently used medications, and is the most common safety reason for the withdrawal of approved drugs from the market. ${ }^{11}$ Idiosyncratic DILI is difficult to predict during pre-clinical and early drug development, and accounts for $11 \%$ of all acute liver failure cases. ${ }^{12}$ Due to the extensive financial losses associated with the removal of an approved drug from the market, and the risk of severe clinical complications of DILI, early identification of compounds that are potentially hepatotoxic is imperative. However, this can be very challenging. In many cases, a liver toxicity signal is not observed in preclinical studies, and first appears during Phase III studies, or even after the drug is approved. Although BSEP inhibition is considered 
one of the contributing factors to the development of cholestatic DILI, even potent BSEP inhibitors cause hepatotoxicity in only a small subset of patients. ${ }^{9,13}$ For example, troglitazone, an antidiabetic medication withdrawn from the market due to DILI, and the metabolite, troglitazone sulfate, are both potent BSEP inhibitors. ${ }^{13,14}$ However, even with potent in vitro BSEP inhibition, the incidence of patients presenting with elevated liver enzymes during the clinical trials of troglitazone was only $1.9 \% .{ }^{15}$ This indicates that there are additional factors that might increase a patient's susceptibility to hepatotoxicity, and numerous candidate gene studies have been conducted to identify genetic factors that may contribute to the development of cholestatic DILI.

A common variant in $A B C B 11$, $(\mathrm{rs} 2287622 ; \mathrm{c} .1331 \mathrm{~T}>\mathrm{C}$; $\mathrm{p} . \mathrm{V} 444 \mathrm{~A})$ has been reported to be associated with an increased risk of developing cholestatic DILI and ICP ${ }^{16}$, as well as with disease progression in hepatitis C. ${ }^{17,18}$ In a trial of patients presenting with cholestatic DILI, the CC genotype in $A B C B 11$ was more common compared to patients without DILI. ${ }^{19}$ Similar associations between patients with the $\mathrm{CC}$ genotype and the risk of contraceptiveinduced cholestasis and ICP also have been reported. ${ }^{8,20}$ However, a similar study in a Japanese population found no association of cholestasis with this variant. ${ }^{21}$ Studies to explain the mechanistic basis for this increased susceptibility to acquired cholestatic syndromes have been unsuccessful. The expression of the variant BSEP is slightly lower in both liver tissue ${ }^{22}$ and in transfected systems. ${ }^{21,23,24}$ However, this variant is extremely common, with an allele frequency of 53\% in African-Americans, 57\% in European Americans, ${ }^{23}$ and $74 \%$ or higher in Mainland Chinese and other Asian populations. ${ }^{25,26}$ Thus, any clinically impactful changes in transporter expression of variant BSEP would place a large portion of the population at risk for DILI and ICP, which is not consistent with clinical observations.

Functional studies have found that the kinetics of taurocholic acid (TCA) transport are similar between the reference and the variant forms of BSEP. ${ }^{19,23}$ TCA is a prototypical bile acid frequently used for in vitro studies because it is readily available radiolabeled. TCA is one of many bile acid species present in humans; each bile acid has a unique toxicity profile and substrate affinity for BSEP. ${ }^{27}$ In humans, glycine conjugated bile acids predominate over taurine conjugated bile acids. ${ }^{28}$ Interestingly, the transport kinetics of additional bile acid substrates such as glycocholic acid (GCA) have not been investigated for this variant. Furthermore, the inhibitory potential of medications for the reference and variant forms of BSEP warrants investigation. A previous study by Ulzurrun et al. evaluated the interaction between therapeutic class/chemical structure of a compound and the p.V444A variant in patients with DILI and found that for certain chemical moieties the association was stronger than others. ${ }^{29}$ However, this interaction between the BSEP variant protein and the inhibiting medication has not been experimentally verified. This study was designed to characterize the function of this common variant by determining the transport kinetics of GCA and evaluating the inhibitory potential of compounds associated with DILI between the reference and variant forms. 


\section{Methods:}

\section{Materials:}

Spodoptera frugiperda 9 (Sf9) cells were obtained from the Tissue Culture Facility at the University of North Carolina-Chapel Hill. Cell culture media (Sf-900 II Serum Free Media) and TaqMan ${ }^{\circledR}$ genotyping supplies (including assay and master mix), were purchased from Thermo Fisher Scientific (Waltham, MA). BSEP antibody for immunoblot analysis was purchased from Abcam (ab140616; Cambridge, MA) and anti-rabbit IgG secondary antibody was purchased from Jackson ImmunoResearch (West Grove, PA). $\left[{ }^{14} \mathrm{C}\right]-$ Glycocholic acid $\left(46.3 \mathrm{mCi} / \mathrm{mmol} ;>97 \%\right.$ radiochemical purity) and $\left[{ }^{3} \mathrm{H}\right]$-taurocholic acid $(9.74 \mathrm{Ci} / \mathrm{mmol} ;>97 \%$ radiochemical purity) were purchased from Perkin Elmer (Waltham, MA). Compounds tested for inhibition studies were purchased from either Fisher Scientific (Thermo Fisher Scientific; Waltham, MA) or Sigma-Aldrich (St. Louis, MO). The scintillation cocktail, Bio-Safe II Complete Counting Cocktail, was purchased from Research Products International (Mt. Prospect, IL).

\section{Generation and characterization of membrane vesicles:}

Baculoviral stocks for the expression of either the p.444V (reference) or p.444A (variant) BSEP were generated previously. ${ }^{19}$ The viral stocks were genotyped using TaqMan ${ }^{\circledR}$ Drug Metabolism Genotyping Assay (Catalog Number 4362691, Thermo Fisher Scientific). DNA was extracted from baculovirus stocks as described in the Easy-DNA ${ }^{\mathrm{TM}}$ Kit Manual (Catalog Number K1800-01, Life Technologies, Carlsbad, CA). The samples were genotyped for the rs2287622 single nucleotide polymorphism (SNP) using a QuantStudio ${ }^{\text {TM }} 6$ Flex System (Applied Biosystems ${ }^{\mathrm{TM}}$ Thermo Fisher Scientific) to confirm the genotype of the baculoviral stocks.

Sf9 cells were infected with the baculoviral stocks and membrane vesicles were generated as described previously. ${ }^{19}$ Briefly, Sf9 cells were grown at $27{ }^{\circ} \mathrm{C}$ and were infected with either the p.444V or p.444A baculovirus for an incubation period of 3 days. Following this incubation period, the cells were harvested and centrifuged to pellet the cells. The supernatant and the cell pellet were reconstituted in TMEP buffer $(50 \mathrm{mM}$ Tris, $50 \mathrm{mM}$ Mannitol, $2 \mathrm{mM}$ EGTA, adjusted to a $\mathrm{pH}$ of 7.0 with $\mathrm{HCl}$ ) containing protease inhibitors. The resulting suspension was homogenized using a glass-teflon homogenizer and centrifuged at $600 \mathrm{xg}$ for $10 \mathrm{~min}$. The supernatant was collected and centrifuged at 100,000 $\mathrm{xg}$ for $1 \mathrm{hr}$ (Beckman Coulter 25,000 RPM). The resulting pellet was resuspended in SMS buffer (50 mM sucrose, $100 \mathrm{mM} \mathrm{KNO}_{3}$ and $10 \mathrm{mM}$ Hepes, adjusted to $\mathrm{pH} 7.4$ using Tris) and the suspension was passed through a $27 \mathrm{G}$ needle to generate membrane vesicles. The protein concentration was determined using a BCA Protein Assay (Thermo Fisher Scientific) and vesicles were aliquoted and stored at $-80{ }^{\circ} \mathrm{C}$. The protein expression of BSEP in the reference and variant vesicles was measured using immunoblot analysis as previously described. ${ }^{30}$

Transport of TCA was measured to characterize the functionality of the generated membrane vesicles using the assay conditions described previously. ${ }^{31}$ The kinetics of GCA transport mediated by reference and variant BSEP were evaluated by incubating membrane vesicles 
(10 $\mu$ g/reaction) for $1 \mathrm{~min}$ with approximately $2 \mu \mathrm{M}$ of $\left[{ }^{14} \mathrm{C}\right]$-GCA along with unlabeled GCA over a range of concentrations $(1,10,25,50,100 \mu \mathrm{M})$ in the presence or absence of 4 $\mathrm{mM}$ ATP. The reaction was stopped by addition of ice-cold buffer, and the samples were filtered through glass fiber filters that were pre-soaked in a solution of unlabeled GCA (1 $\mathrm{mM})$. The filters were dissolved in scintillation cocktail and the amount of radioactivity in the vesicles was measured by scintillation counting. Radioactivity in the vesicles incubated without ATP was subtracted from radioactivity in the vesicles incubated with ATP to determine ATP-dependent transport. Nonlinear regression analysis was used to estimate the $\mathrm{V}_{\max }$ and $\mathrm{K}_{\mathrm{m}}$ values using GraphPad Prism version 7.04 (La Jolla, California).

\section{Inhibition Studies:}

Previous studies reported a possible association between the pharmacological drug class/ chemical moieties and susceptibility to cholestasis in patients expressing the variant protein. ${ }^{29}$ A panel of these compounds was tested as inhibitors of both TCA $(2 \mu \mathrm{M}$; incubation time of $2 \mathrm{~min}$ ) and GCA transport (10 $\mu \mathrm{M}$; incubation time of $1 \mathrm{~min})$ mediated by reference and variant BSEP to identify any substrate specific differences in inhibitory potential. Inhibition studies were conducted at an inhibitor concentration of $100 \mu \mathrm{M}$. Transport activity in the presence of inhibitors was normalized to transport activity measured in the control (DMSO) group for both the reference and variant BSEP to obtain the relative activity (\% Activity).

Additional inhibition studies were conducted to further characterize interactions between DILI associated compounds and the p.V444A variant. TCA was used as the substrate in these studies because it is the prototypical BSEP substrate, and has been used in previous studies to identify inhibitors. ${ }^{10,32}$ Compounds tested as inhibitors included causative cholestatic DILI medications as identified through the Drug-Induced Liver Injury Network (DILIN) registry, a prospective registry of patients with DILI established by the National Institute of Diabetes and Digestive and Kidney Diseases (NIDDK). ${ }^{33}$ Compounds that had been tested in the inhibition study with TCA and GCA were excluded from this analysis (amoxicillin-clavulanate, dipyridamole, isoniazid, regorafenib). Forty-six compounds associated with cholestatic DILI were tested as TCA inhibitors. Test compounds were dissolved in DMSO and inhibition studies were conducted at a concentration of $100 \mu \mathrm{M}$ in a high-throughput assay, as described previously. ${ }^{34}$ Compounds that had a fold-change of $<0.5$ or $>2$ between the reference and variant protein were selected for further investigations to determine $\mathrm{IC}_{50}$ values. All inhibition studies were conducted in triplicate; the experiments using TCA as a substrate were conducted in one experiment. Inhibition studies that utilized GCA as a substrate were conducted in two independent experiments $(n=2)$ because the inhibition of BSEP-mediated GCA transport by these compounds had not been reported previously.

\section{Statistical analysis:}

An extra sum-of-squares $F$ test was used to compare independent fits that described the kinetics of GCA transport for the reference and variant forms of BSEP to a global fit curve that described both sets of observations. Statistically significant differences between reference and variant BSEP in the transport of TCA or GCA were tested using a two-way ANOVA with a Sidak test to correct for multiple-comparisons. 


\section{Results:}

Expression of both reference and variant BSEP in Sf9 derived membrane vesicles determined by western blot analysis were similar (Figure 1). TCA transport for both reference and variant forms of BSEP was comparable(Supplementary Figure 1). The transport kinetics of GCA for reference and variant BSEP followed Michaelis-Menten kinetics with $\mathrm{V}_{\max }$ values of $1132 \pm 246 \mathrm{vs} .959 \pm 256 \mathrm{pmol} / \mathrm{min} / \mathrm{mg}$ protein, respectively, and $\mathrm{K}_{\mathrm{m}}$ values of $32.74 \pm 18.25$ vs. $45.70 \pm 25.52 \mu \mathrm{M}$, respectively. GCA transport was not significantly different between reference and variant BSEP $(\mathrm{p}=0.0784)$ over the range of concentrations studied.

Under all conditions tested, BSEP was inhibited strongly (defined here as greater than $75 \%$ inhibition) by dipyridamole, erythromycin, and ketoconazole (Figure 2 and Supplementary Table 1). A slight potentiation of reference and variant BSEP-mediated TCA and GCA transport was observed with amoxicillin-clavulanate. There were no statistically significant differences in \% Activity in the presence of the compounds tested for either substrate (TCA or GCA) between reference and variant BSEP.

Of the DILIN compounds tested in the TCA inhibition study, ten compounds exhibited a greater than 50\% reduction in TCA transport by reference BSEP (Figure 3). These compounds included amiodarone, torimefene, the clinically used combination of norgestimate and ethinyl estradiol, chlorothiazide, cefazolin, allopurinol, and acyclovir. The mean \% Activity of reference and variant BSEP in the presence of the tested inhibitors (100 $\mu \mathrm{M}$ ) was within a 2-fold difference, except for sulindac (Figure 3 and Supplementary Table 2). However, when sulindac was tested over a range of concentrations (up to $200 \mu \mathrm{M}$ ), the $\mathrm{IC}_{50}$ values were similar between the reference $(109.3 \pm 25.4 \mu \mathrm{M})$ and variant $(136.2 \pm 25.1$ $\mu \mathrm{M}) \mathrm{BSEP}$.

\section{Discussion}

The functional impact of p.V444A, a common variant in BSEP, was evaluated in this study to identify a mechanistic explanation for the reported association of this SNP with acquired cholestasis. Potential changes in the substrate affinity were first examined by measuring the transport kinetics of GCA. Then, the inhibition of reference and variant BSEP by cholestatic compounds was investigated to test whether the two forms of the protein differ in their susceptibility to drug inhibition, thereby providing functional data supporting the association with DILI.

Variant (444A) BSEP has been reported to be associated with an increased risk of both cholestatic DILI and ICP in multiple studies. In a candidate gene study of patients with DILI, the CC genotype (444A) in $A B C B 11$ was more common in patients with DILI than in the control group. ${ }^{19}$ Similar findings also were reported in European and Caucasian populations,${ }^{29}$ however, no statistically significant association was reported in studies conducted in Chinese and Japanese cohorts. ${ }^{21,35}$ The 444A variant of BSEP also has been identified as a risk factor for the development of ICP, with the variant being more frequent in ICP patients when compared to pregnant controls. ${ }^{8,20,36}$ Dixon et al. reported an increased 
risk of ICP in subjects with the 444A variant, especially those who were homozygous for this BSEP variant. However, structural analysis utilizing homology modeling with the bacterial transporter protein Sav1866 found no molecular basis for the association reported with the p.V444A variant. ${ }^{8}$ Based on in silico tools that are used to predict the functional consequences of SNPs, the p.V444A variant is predicted to be benign. ${ }^{25}$

These predictions are in line with in vitro results demonstrating that the transport of TCA by reference and variant BSEP are identical. ${ }^{19,23}$ However, other bile acids, including GCA, are also transported by BSEP and exhibit different transport kinetics. ${ }^{3,4,27}$ In the present study, the transport of GCA was evaluated to test the hypothesis that this bile acid, which is one of the predominant bile acids present in humans ${ }^{28}$ with lower affinity for BSEP, ${ }^{27,37}$ exhibits substrate specific differences due to the SNP. The transport kinetics of GCA over the range of concentrations studied was not statistically different between reference and variant BSEP, indicating that there are no significant changes in GCA transport due to this variant. The $\mathrm{K}_{\mathrm{m}}$ estimates in the present study are similar to those previously reported for reference BSEPexpressing membrane vesicles. ${ }^{38,39}$ It was demonstrated previously that there was no difference in the transport kinetics of TCA between the two variants. ${ }^{19}$ This kinetic similarity is not surprising, since the amino acid at position 444 is located in the large cytoplasmic domain of BSEP linking the two transmembrane domains. ${ }^{19}$

The p.444A variant of BSEP is associated with acquired cholestatic DILI and ICP, conditions where cholestasis is precipitated either due to a medication or an endogenous hormone. Therefore, it is essential to identify any potentially different drug-transporter interactions between reference and variant BSEP. Compounds tested in the first set were those that had been reported previously to have statistical associations with variant BSEP. ${ }^{29}$ The inhibition of both TCA and GCA transport was tested to screen for any potential substrate- and/or variant-specific drug inhibition. The results from the inhibition screening using TCA as the substrate were in agreement with previously published $\mathrm{IC}_{50}$ values for reference BSEP. ${ }^{13}$ For example, compounds that strongly inhibited BSEP activity in our study (dipyridamole, erythromycin, ketoconazole) were reported to be potent inhibitors. ${ }^{13}$ Additionally, compounds that did not inhibit BSEP activity in our study (including isoniazid, ketorolac, levofloxacin) also have been classified as non-inhibitors. ${ }^{13}$ For the tested compounds, no differences in the inhibition of TCA or GCA were observed between reference and variant BSEP. A similar interaction of the inhibitors with TCA and GCA was likely because substrate concentrations well below the respective $K_{m}$ values were used. ${ }^{38,39}$ Follow-up inhibition studies utilized TCA as the substrate and tested cholestatic compounds from the DILIN registry using a high-throughput method. Although inhibition of sulindac was statistically different in the high-throughput screen, when sulindac was tested over a range of concentrations, the $\mathrm{IC}_{50}$ values were similar for both reference and variant $\mathrm{BSEP}$. The results from the inhibition studies conducted here do not demonstrate any variant specific drug interactions with BSEP that would explain the association with DILI.

Although a thorough characterization of the functional activity of this common BSEP variant was conducted, there are a few limitations that should be acknowledged. Membrane vesicles expressing the transporter of interest are an important in vitro tool to characterize transporter function, but they do not reflect the complex and dynamic processes that regulate 
BSEP expression and function in hepatocytes. ${ }^{19}$ Membrane vesicles lack the machinery to allow the study of localization and trafficking, which could be altered in this particular BSEP variant. Although this study utilized TCA and GCA as substrates, potential variantspecific functional changes in the transport kinetics of more toxic bile acids cannot be ruled out. However, such changes are unlikely due to the location of the variant amino acid in the protein, as discussed above, and the comparable affinities between reference and variant BSEP for most bile salts tested so far. ${ }^{39}$ Additionally, there could be other rare variants in $A B C B 11$ that alter function and localization and are yet to be identified. The low frequency of DILI and ICP, and the high frequency of the variant BSEP (50-70\% depending on the ethnic population), are consistent with our findings that this variant does not have major alterations in BSEP function.

In this functional evaluation of the common p.V444A variant of BSEP, no differences in the transport of the bile acid substrate GCA were observed compared with reference BSEP. Additionally, no substrate- or genotype-specific differences were observed in the inhibition potential of medications associated with cholestatic DILI. Under the conditions tested in these studies, no significant functional differences between variant and reference BSEP were identified that would explain the association of this variant with DILI and ICP.

\section{Supplementary Material}

Refer to Web version on PubMed Central for supplementary material.

\section{Acknowledgements}

The authors declare no competing financial interests. Financial support was provided by the National Institute of General Medical Sciences of the National Institutes of Health under Award Numbers T32GM086330 (Izna Ali), R01GM041935 and R35GM122576 (Kim L.R Brouwer). The content is solely the responsibility of the authors and does not necessarily represent the official views of the National Institutes of Health. Dr. Kim L.R. Brouwer is coinventor of the sandwich-cultured hepatocyte technology for quantification of biliary excretion (B-CLEAR ${ }^{\circledR}$ ) and related technologies, which have been licensed exclusively to Qualyst Transporter Solutions, recently acquired by BioIVT. Dr. Bruno Stieger received support from the SNF Center of Competence in Research TransCure (University of Berne, Switzerland). The assistance of Dr. Dong Fu and Chitra Saran (immunoblot analysis), Jacqueline Bezencon (cell culture), Rebecca Rementer (genotyping), and Yi Zeng (transport assays) is gratefully acknowledged. We also acknowledge Dr. Liz Cirulli for providing the list of compounds associated with cholestasis from the Drug-Induced Liver Injury Network (DILIN) registry. The authors would like to thank Dr. Paul Watkins for his helpful suggestions and scientific guidance during the preparation of this manuscript. This work was presented, in part, at the following conferences: 2018 American Association of Pharmaceutical Scientists (AAPS) Workshop on Drug Transporters and the 2018 AAPS Annual Meeting.

\section{References}

(1). Trauner M; Claudel T; Fickert P; Moustafa T; Wagner M Bile Acids as Regulators of Hepatic Lipid and Glucose Metabolism. Dig. Dis 2010, 28 (1), 220-224. [PubMed: 20460915]

(2). Perez M-J; Briz O Bile-Acid-Induced Cell Injury and Protection. World J. Gastroenterol 2009, 15 (14), 1677-1689. [PubMed: 19360911]

(3). Noé J; Stieger B; Meier PJ Functional Expression of the Canalicular Bile Salt Export Pump of Human Liver. Gastroenterology 2002, 123 (5), 1659-1666. [PubMed: 12404240]

(4). Byrne JA; Strautnieks SS; Mieli-Vergani G; Higgins CF; Linton KJ; Thompson RJ The Human Bile Salt Export Pump: Characterization of Substrate Specificity and Identification of Inhibitors. Gastroenterology 2002, 123 (5), 1649-1658. [PubMed: 12404239] 
(5). Kubitz R; Keitel V; Scheuring S; Kohrer K; Haussinger D Benign Recurrent Intrahepatic Cholestasis Associated With Mutations of the Bile Salt Export Pump. J. Clin. Gastroenterol 2006, 40 (2), 171-175. [PubMed: 16394881]

(6). Jansen PL; Müller M The Molecular Genetics of Familial Intrahepatic Cholestasis. Gut 2000, 47 (1), 1-5. [PubMed: 10861251]

(7). Dixon PH; Sambrotta M; Chambers J; Taylor-Harris P; Syngelaki A; Nicolaides K; Knisely AS; Thompson RJ; Williamson C An Expanded Role for Heterozygous Mutations of ABCB4, ABCB11, ATP8B1, ABCC2 and TJP2 in Intrahepatic Cholestasis of Pregnancy. Sci. Rep 2017, 7 (1), 11823. [PubMed: 28924228]

(8). Dixon PH; van Mil SWC; Chambers J; Strautnieks S; Thompson RJ; Lammert F; Kubitz R; Keitel V; Glantz A; Mattsson L-A; et al. Contribution of Variant Alleles of ABCB11 to Susceptibility to Intrahepatic Cholestasis of Pregnancy. Gut 2009, 58 (4), 537-544. [PubMed: 18987030]

(9). Dawson S; Stahl S; Paul N; Barber J; Kenna JG In Vitro Inhibition of the Bile Salt Export Pump Correlates with Risk of Cholestatic Drug-Induced Liver Injury in Humans. Drug Metab. Dispos 2011, 40 (1).

(10). Morgan RE; Trauner M; van Staden CJ; Lee PH; Ramachandran B; Eschenberg M; Afshari CA; Qualls CW; Lightfoot-Dunn R; Hamadeh HK Interference with Bile Salt Export Pump Function Is a Susceptibility Factor for Human Liver Injury in Drug Development. Toxicol. Sci 2010, 118 (2), 485-500. [PubMed: 20829430]

(11). Onakpoya IJ; Heneghan CJ; Aronson JK Post-Marketing Withdrawal of 462 Medicinal Products Because of Adverse Drug Reactions: A Systematic Review of the World Literature. BMC Med 2016, 14, 10. [PubMed: 26843061]

(12). Leise MD; Poterucha JJ; Talwalkar JA Drug-Induced Liver Injury. Mayo Clin. Proc 2014, 89 (1), 95-106. [PubMed: 24388027]

(13). Morgan RE; van Staden CJ; Chen Y; Kalyanaraman N; Kalanzi J; Dunn RT; Afshari CA; Hamadeh HK; G., S.; W., B. A Multifactorial Approach to Hepatobiliary Transporter Assessment Enables Improved Therapeutic Compound Development. Toxicol. Sci 2013, 136 (1), 216-241. [PubMed: 23956101]

(14). Funk C; Ponelle C; Scheuermann G; Pantze M Cholestatic Potential of Troglitazone as a Possible Factor Contributing to Troglitazone-Induced Hepatotoxicity: In Vivo and in Vitro Interaction at the Canalicular Bile Salt Export Pump (Bsep) in the Rat. Mol. Pharmacol 2001, 59 (3), 627-635. [PubMed: 11179459]

(15). Watkins PB; Whitcomb RW Hepatic Dysfunction Associated with Troglitazone. N. Engl. J. Med 1998, 338 (13), 916-917. [PubMed: 9518284]

(16). Pauli-Magnus C; Meier P; Stieger B Genetic Determinants of Drug-Induced Cholestasis and Intrahepatic Cholestasis of Pregnancy. Semin. Liver Dis 2010, 30 (02), 147-159. [PubMed: 20422497]

(17). Iwata R; Baur K; Stieger B; Mertens JC; Daly AK; Frei P; Braun J; Vergopoulos A; Stickel F; Sabrane K; et al. A Common Polymorphism in the ABCB11 Gene Is Associated with Advanced Fibrosis in Hepatitis C but Not in Non-Alcoholic Fatty Liver Disease. Clin. Sci. (Lond) 2011, 120 (7), 287-296. [PubMed: 20883210]

(18). Stieger B; Geier A Genetic Variations of Bile Salt Transporters as Predisposing Factors for DrugInduced Cholestasis, Intrahepatic Cholestasis of Pregnancy and Therapeutic Response of Viral Hepatitis. Expert Opin. Drug Metab. Toxicol 2011, 7 (4), 411-425. [PubMed: 21320040]

(19). Lang C; Meier Y; Stieger B; Beuers U; Lang T; Kerb R; Kullak-Ublick GA; Meier PJ; PauliMagnus C Mutations and Polymorphisms in the Bile Salt Export Pump and the Multidrug Resistance Protein 3 Associated with Drug-Induced Liver Injury. Pharmacogenet. Genomics 2007, 17 (1), 47-60.

(20). Meier Y; Zodan T; Lang C; Zimmermann R; Kullak-Ublick GA; Meier PJ; Stieger B; PauliMagnus C Increased Susceptibility for Intrahepatic Cholestasis of Pregnancy and ContraceptiveInduced Cholestasis in Carriers of the 1331T >C Polymorphism in the Bile Salt Export Pump. World J. Gastroenterol 2008, 14 (1), 38-45. [PubMed: 18176959]

(21). Kagawa T; Hirose S; Arase Y; Oka A; Anzai K; Tsuruya K; Shiraishi K; Orii R; Ieda S; Nakazawa T; et al. No Contribution of the ABCB11 p.444A Polymorphism in Japanese Patients 
with Drug-Induced Cholestasis. Drug Metab. Dispos 2015, 43 (5), 691-697. [PubMed: 25713208]

(22). Meier Y; Pauli-Magnus C; Zanger UM; Klein K; Schaeffeler E; Nussler AK; Nussler N; Eichelbaum M; Meier PJ; Stieger B Interindividual Variability of Canalicular ATP-BindingCassette (ABC)-Transporter Expression in Human Liver. Hepatology 2006, 44 (1), 62-74. [PubMed: 16799996]

(23). Ho RH; Leake BF; Kilkenny DM; Meyer Zu Schwabedissen HE; Glaeser H; Kroetz DL; Kim RB Polymorphic Variants in the Human Bile Salt Export Pump (BSEP; ABCB11): Functional Characterization and Interindividual Variability. Pharmacogenet. Genomics 2010, 20 (1), 45-57.

(24). Byrne JA; Strautnieks SS; Ihrke G; Pagani F; Knisely AS; Linton KJ; Mieli-Vergani G; Thompson RJ Missense Mutations and Single Nucleotide Polymorphisms in ABCB11 Impair Bile Salt Export Pump Processing and Function or Disrupt Pre-Messenger RNA Splicing. Hepatology 2009, 49 (2), 553-567. [PubMed: 19101985]

(25). Hu G; He P; Liu Z; Chen Q; Zheng B; Zhang Q Diagnosis of ABCB11 Gene Mutations in Children with Intrahepatic Cholestasis Using High Resolution Melting Analysis and Direct Sequencing. Mol. Med. Rep 2014, 10 (3), 1264-1274. [PubMed: 24969679]

(26). Chen H-L; Liu Y-J; Su Y-N; Wang N-Y; Wu S-H; Ni Y-H; Hsu H-Y; Wu T-C; Chang M-H Diagnosis of BSEP/ABCB11 Mutations in Asian Patients with Cholestasis Using Denaturing High Performance Liquid Chromatography. J. Pediatr 2008, 153 (6), 825-832.e2. [PubMed: 18692205]

(27). Hayashi H; Takada T; Suzuki H; Onuki R; Hofmann AF; Sugiyama Y Transport by Vesicles of Glycine- and Taurine-Conjugated Bile Salts and Taurolithocholate 3-Sulfate: A Comparison of Human BSEP with Rat Bsep. Biochim. Biophys. Acta 2005, 1738 (1-3), 54-62. [PubMed: 16332456]

(28). Schadt HS; Wolf A; Pognan F; Chibout S-D; Merz M; Kullak-Ublick GA Bile Acids in Drug Induced Liver Injury: Key Players and Surrogate Markers. Clin. Res. Hepatol. Gastroenterol 2016, 40 (3), 257-266. [PubMed: 26874804]

(29). Ulzurrun E; Stephens C; Crespo E; Ruiz-Cabello F; Ruiz-Nuñez J; Saenz-López P; MorenoHerrera I; Robles-Díaz M; Hallal H; Moreno-Planas JM; et al. Role of Chemical Structures and the 1331T >C Bile Salt Export Pump Polymorphism in Idiosyncratic Drug-Induced Liver Injury. Liver Int 2013, 33 (9), 1378-1385. [PubMed: 23701583]

(30). Guo C; LaCerte C; Edwards JE; Brouwer KR; Brouwer KLR Farnesoid X Receptor Agonists Obeticholic Acid and Chenodeoxycholic Acid Increase Bile Acid Efflux in Sandwich-Cultured Human Hepatocytes: Functional Evidence and Mechanisms. J Pharmacol. Exp. Ther 2018, 365 (2), 413-421. [PubMed: 29487110]

(31). Slizgi JR; Lu Y; Brouwer KR; St. Claire RL; Freeman KM; Pan M; Brock WJ; Brouwer KLR Inhibition of Human Hepatic Bile Acid Transporters by Tolvaptan and Metabolites: Contributing Factors to Drug-Induced Liver Injury? Toxicol. Sci 2016, 149 (1), 237-250. [PubMed: 26507107]

(32). Pedersen JM; Matsson P; Bergström CAS; Hoogstraate J; Norén A; LeCluyse EL; Artursson P Early Identification of Clinically Relevant Drug Interactions with the Human Bile Salt Export Pump (BSEP/ABCB11). Toxicol. Sci 2013, 136 (2), 328-343. [PubMed: 24014644]

(33). Fontana RJ; Watkins PB; Bonkovsky HL; Chalasani N; Davern T; Serrano J; Rochon J; DILIN Study Group. Drug-Induced Liver Injury Network (DILIN) Prospective Study: Rationale, Design and Conduct. Drug Saf 2009, 32 (1), 55-68. [PubMed: 19132805]

(34). Köck K; Ferslew BC; Netterberg I; Yang K; Urban TJ; Swaan PW; Stewart PW; Brouwer KLR Risk Factors for Development of Cholestatic Drug-Induced Liver Injury: Inhibition of Hepatic Basolateral Bile Acid Transporters Multidrug Resistance-Associated Proteins 3 and 4. Drug Metab. Dispos 2014, 42 (4), 665-674. [PubMed: 24154606]

(35). Chen R; Wang J; Tang S; Zhang Y; Lv X; Wu S; Yang Z; Xia Y; Chen D; Zhan S Role of Polymorphic Bile Salt Export Pump (BSEP, ABCB11) Transporters in Anti-Tuberculosis DrugInduced Liver Injury in a Chinese Cohort. Sci. Rep 2016, 6 (1), 27750 10.1038/srep27750. [PubMed: 27293027]

(36). Piątek K; Kurzawińska G; Magiełda J; Drews K; Barlik M; Malewski Z; Ożarowski M; Maciejewska M; Seremak-Mrozikiewicz A The Role of ABC Transporters' Gene Polymorphism 
in the Etiology of Intrahepatic Cholestasis of Pregnancy. Ginekol. Pol 2018, 89 (7), 393-397. [PubMed: 30091450]

(37). van Beusekom CD; van den Heuvel JJ; Koenderink JB; Schrickx JA; Russel FG The Feline Bile Salt Export Pump: A Structural and Functional Comparison with Canine and Human Bsep/BSEP. BMC Vet. Res 2013, 9 (1), 259. [PubMed: 24359682]

(38). Kis E; Ioja E; Nagy T; Szente L; Herédi-Szabó K; Krajcsi P Effect of Membrane Cholesterol on BSEP/Bsep Activity: Species Specificity Studies for Substrates and Inhibitors. Drug Metab. Dispos 2009, 37 (9), 1878-1886. [PubMed: 19520776]

(39). Stieger B The Role of the Sodium-Taurocholate Cotransporting Polypeptide (NTCP) and of the Bile Salt Export Pump (BSEP) in Physiology and Pathophysiology of Bile Formation. In Handbook of Experimental Pharmacology; Fromm M, Kim R, Eds.; Springer, Berlin, Heidelberg, 2011; pp 205-259. 
A

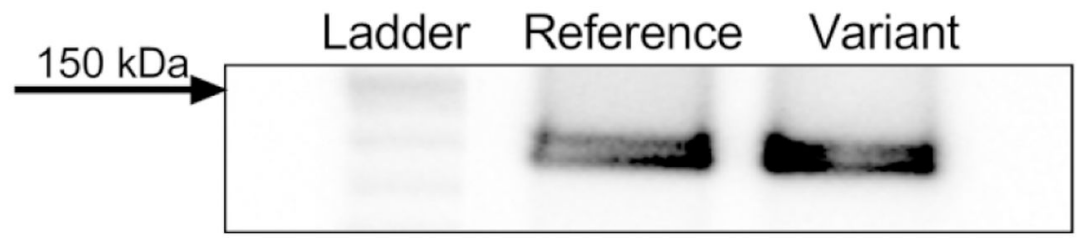

B

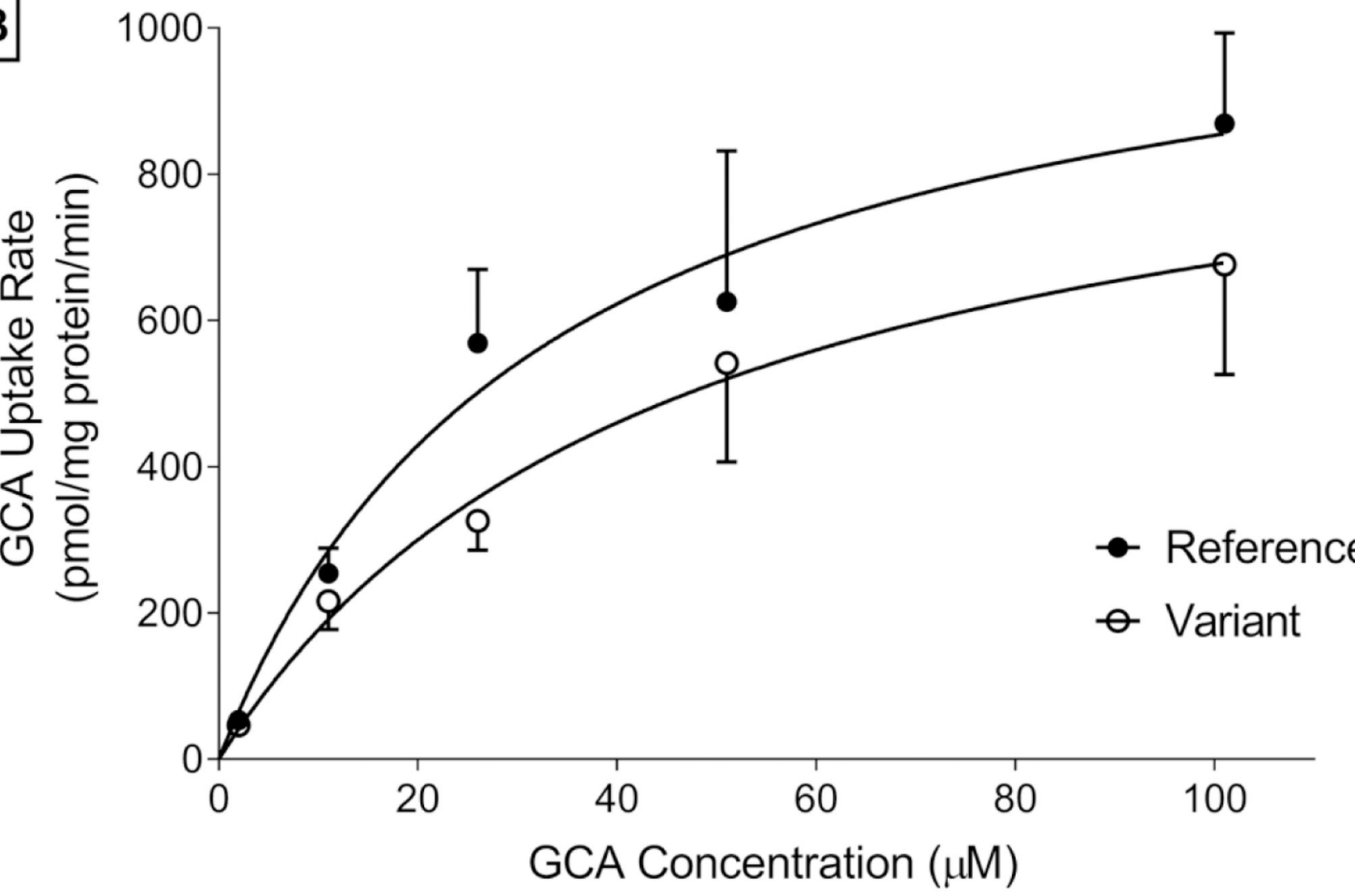

Figure 1: Comparison of GCA transport kinetics mediated by reference and variant BSEP.

[A] BSEP levels were similar in reference and variant membrane vesicles based on western blot analysis. [B] Membrane vesicles were incubated with a range of GCA concentrations in the presence or absence of $4 \mathrm{mM}$ ATP for $1 \mathrm{~min}$. The difference in curves for ATPdependent transport of GCA by the reference and variant protein was tested for statistical significance using an Extra sum-of-squares $\mathrm{F}$ test. Mean \pm SEM ( $\mathrm{n}=3$ in triplicate). 


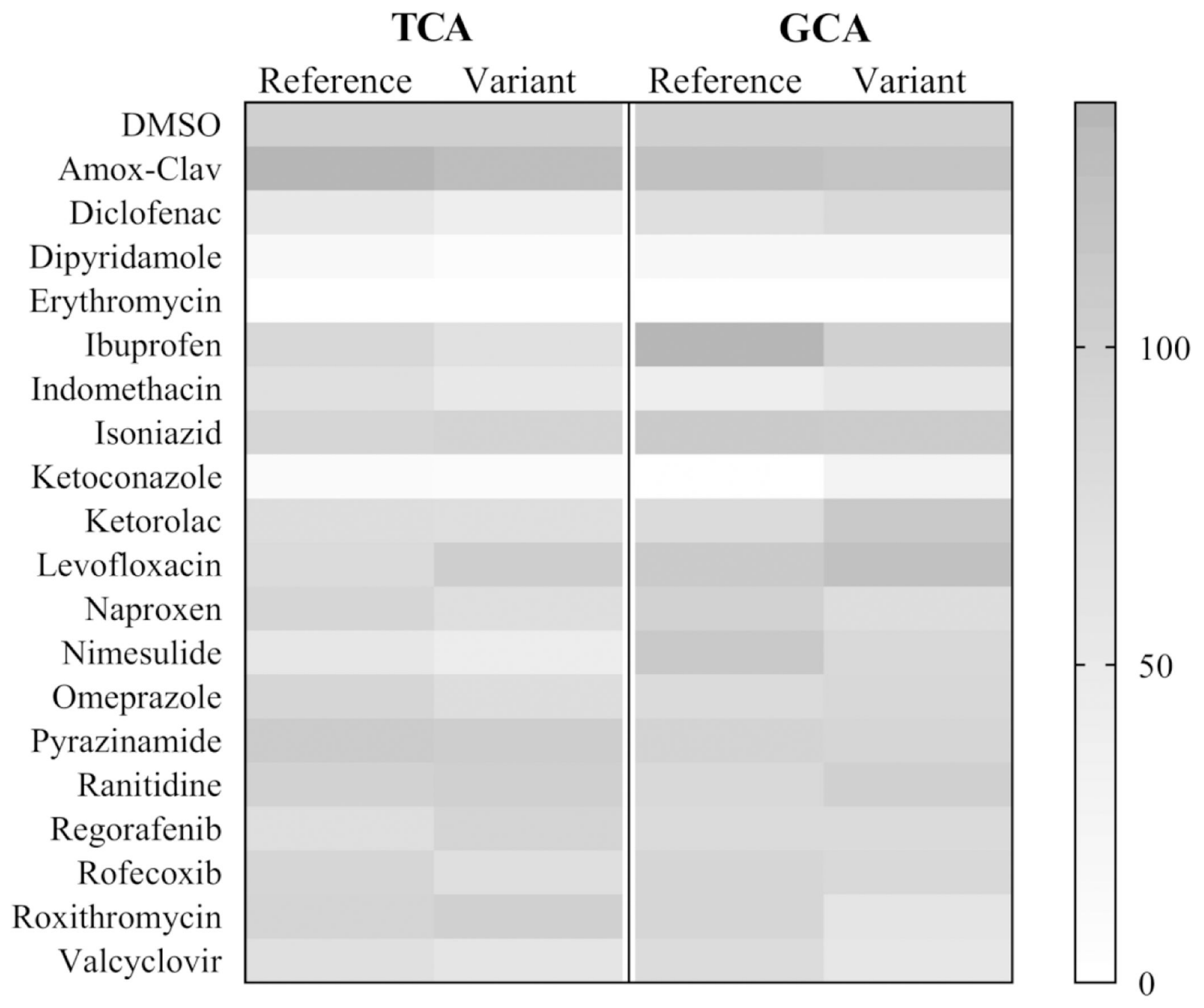

Figure 2: Inhibition of TCA and GCA transport mediated by reference and variant BSEP.

Mean values (\% Activity based on the gray-scale bar on the right where $100 \%$ indicates no inhibition and $0 \%$ indicates complete inhibition) were normalized to DMSO (Mean values from $n=1-2$ experiments conducted in triplicate). 


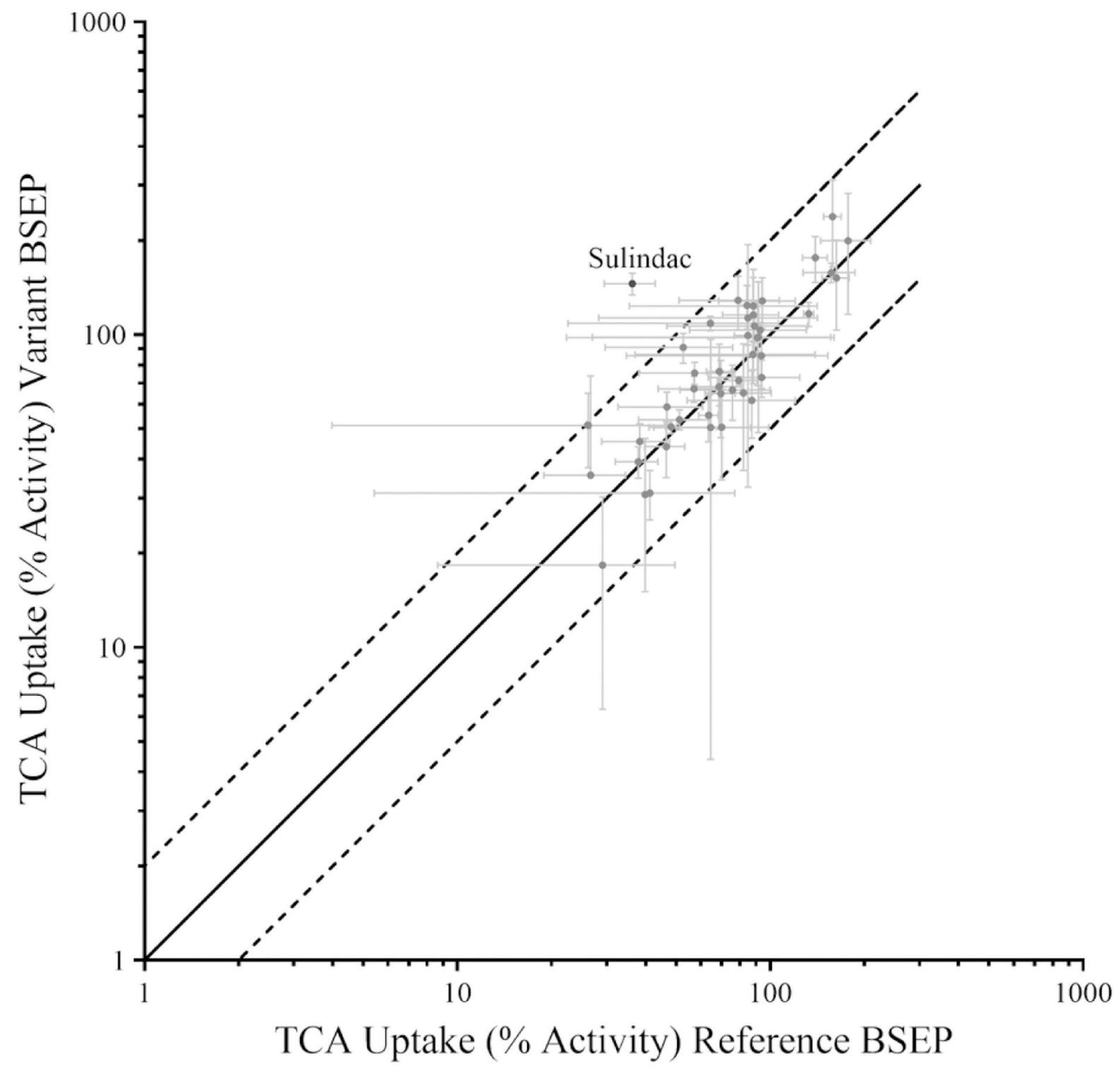

Figure 3: Inhibition of TCA transport mediated by reference and variant BSEP.

Compounds from the DILIN Registry were tested at a concentration of $100 \mu \mathrm{M}$. Data are expressed as \% Activity normalized to DMSO, where $100 \%$ indicates no inhibition. (Mean \pm SD from $n=1$ experiment conducted in triplicate). 